\title{
Assessing the temperature dependence of narrow-band Raman water vapor lidar measurements: a practical approach
}

\author{
David N. Whiteman, ${ }^{1, \star}$ Demetrius D. Venable, ${ }^{2}$ Monique Walker, ${ }^{2}$ Martin Cadirola, ${ }^{3}$ \\ Tetsu Sakai, ${ }^{4}$ and Igor Veselovskii ${ }^{5}$ \\ 'NASA/GSFC, Greenbelt, Maryland 20771, USA \\ ${ }^{2}$ Howard University, Washington, District of Columbia 20059, USA \\ ${ }^{3}$ Ecotronics, LLC, Clarksburg, Maryland 20871, USA \\ ${ }^{4}$ Meteorological Research Institute, Tsukuba, Ibaraki 305-0052, Japan \\ ${ }^{5}$ Physics Instrumentation Center, Troitsk, Russia \\ *Corresponding author: david.n.whiteman@ @asa.gov \\ Received 22 February 2013; accepted 19 April 2013; \\ posted 28 June 2013 (Doc. ID 185825); published 24 July 2013
}

\begin{abstract}
Narrow-band detection of the Raman water vapor spectrum using the lidar technique introduces a concern over the temperature dependence of the Raman spectrum. Various groups have addressed this issue either by trying to minimize the temperature dependence to the point where it can be ignored or by correcting for whatever degree of temperature dependence exists. The traditional technique for performing either of these entails accurately measuring both the laser output wavelength and the water vapor spectral passband with combined uncertainty of approximately $0.01 \mathrm{~nm}$. However, uncertainty in interference filter center wavelengths and laser output wavelengths can be this large or larger. These combined uncertainties translate into uncertainties in the magnitude of the temperature dependence of the Raman lidar water vapor measurement of $3 \%$ or more. We present here an alternate approach for accurately determining the temperature dependence of the Raman lidar water vapor measurement. This alternate approach entails acquiring sequential atmospheric profiles using the lidar while scanning the channel passband across portions of the Raman water vapor $Q$-branch. This scanning is accomplished either by tilt-tuning an interference filter or by scanning the output of a spectrometer. Through this process a peak in the transmitted intensity can be discerned in a manner that defines the spectral location of the channel passband with respect to the laser output wavelength to much higher accuracy than that achieved with standard laboratory techniques. Given the peak of the water vapor signal intensity curve, determined using the techniques described here, and an approximate knowledge of atmospheric temperature, the temperature dependence of a given Raman lidar profile can be determined with accuracy of $0.5 \%$ or better. A Mathematica notebook that demonstrates the calculations used here is available from the lead author. (C) 2013 Optical Society of America

OCIS codes: (010.3640) Lidar; (300.6450) Spectroscopy, Raman.

http://dx.doi.org/10.1364/AO.52.005376
\end{abstract}

\section{Introduction}

Raman lidar is a well-established technique for probing water vapor in the atmosphere dating from

$1559-128 \mathrm{X} / 13 / 225376-09 \$ 15.00 / 0$

(C) 2013 Optical Society of America the first nighttime measurements of Cooney [1] and Melfi [2] to the current capability of day and night measurements offered by numerous lidar sytems [3-8]. Although very useful Raman lidar measurements of water vapor and other species have been made with the solar blind technique [9], the narrowband, narrow field of view (NBNFŌV) approach, 
which makes use of high UV laser energy, narrow spectral and spatial filtering and a combination of analog and photon counting data acquisition [3,4], has become the most popular Raman lidar technique for measuring water vapor. The reason for the popularity of the NBNFOV technique is that it permits useful daytime measurements to be performed without limiting the upper range of nighttime measurements as is the case when using the solar blind technique.

It was first noted by Sherlock et al. [10], however, that the use of narrow band detection of the water vapor spectrum introduces a concern about the temperature dependence of the Raman lidar water vapor measurement. That work was based on a Raman scattering model that did not include anisotropic scattering. Fundamental theoretical work in the spectroscopy of the Raman $\mathrm{OH}$ stretch region, performed by Avila et al. [11] and that built upon the work of Murphy [12,13] and Fernández-Sánchez $[14,15]$, included anisotropic scattering explicitly. This advance in Raman scattering modeling permitted Whiteman $[16,17]$ to demonstrate an accurate correction technique for this temperature dependence of water vapor using a modified version of the lidar equation that explicitly contained the temperature dependence as a multiplier of the traditional lidar equation. It became clear from that work, however, that laser and interference filter characteristics had to be known with quite high accuracy to optimally implement the correction. Whiteman et al. applied this formulation to the correction of a wet bias observed during the International $\mathrm{H}_{2} \mathrm{O}$ Project (IHOP) field campaign [4] and Atmospheric Infrared Sounder (AIRS) validation measurements [18] that significantly improved the upper tropospheric measurements of their scanning Raman lidar. Application of the technique, however, was performed using high resolution wavemeter measurements of the output spectrum of the laser, as well as mercury lamp calibrated Fourier transform spectrometer (FTS) and spectrophotometer measurements of the interference filter. It was also necessary to assess any deviations from pure normal positioning of the interference filter.

Given that discrepancies between manufacturer's filter transmission curves and high resolution FTS measurements have shown differences typically of $0.01-0.02 \mathrm{~nm}$, but at times as large as $0.05 \mathrm{~nm}$ [18], implementation of an accurate solution for this temperature dependence requires very careful laboratory work whether the desire is either to minimize the temperature dependence or to correct for it.

Because of the difficulty of determining the temperature dependence of Raman lidar measurements of water vapor using these previously described techniques, a new approach was conceived and implemented in 2007 as part of the NASA/GSFC ALVICE mobile lidar system [5]. That approach entails tilt-tuning [19] interference filters for both the Raman water vapor and nitrogen channels while the filters remain in the optical train and acquiring a sequential set of atmospheric profiles. Although the discussion here will focus mainly on the use of interference filters, the same approach can be implemented using a spectrometer-based system by scanning the output of the spectrometer. Also, in this article we consider only the temperature dependence of the Raman water vapor measurement. The measurement of the water vapor mixing ratio from Raman lidar also requires measuring the Raman nitrogen signal, which possesses some temperature dependence as well. But, provided that narrow-band filters with widths in the range of $0.2-0.3 \mathrm{~nm}$ are used, the temperature dependence of the Raman nitrogen measurement is less than $0.5 \%$ over the range of normal atmospheric temperatures [16] and thus will not be considered here. Note, however, that the use of wide-band filters in the measurement of the Raman nitrogen signal can increase the temperature dependence of the measurement into the $1 \%-2 \%$ range [16]. We will describe the new approach proposed here in more detail after reviewing the temperature-dependent lidar equation [ㅌ, $\underline{17}]$.

\section{Temperature-Dependent Lidar Equation}

The idea behind this equation, which was motivated by the work of Sherlock et al. [10], is to include the temperature dependence of the lidar equation into a separate, multiplicative factor that can be assessed separately from the elements of the normal, temperature-independent, lidar equation. That factor is referred to as $F_{H}$ below.

The temperature-dependent lidar equation can be written

$$
\begin{aligned}
P\left(r, \lambda_{L}, \lambda_{H}, \Delta \lambda_{H}\right)= & P_{0} \frac{c \tau}{2} \frac{A}{r^{2}} \eta\left(\lambda_{H}\right) O(r) F_{H}(T) \beta_{\pi}^{H}\left(\lambda_{L}, r\right) \\
& \times \exp \left(-\int_{0}^{r}\left[\alpha\left(\lambda_{L}, r^{\prime}\right)+\alpha\left(\lambda_{H}, r^{\prime}\right)\right] \mathrm{d} r^{\prime}\right) \\
& +B\left(r, \lambda_{H}, \Delta \lambda_{H}\right)
\end{aligned}
$$

where

1. $P\left(r, \lambda_{L}, \lambda_{H}, \Delta \lambda_{H}\right)$ is the return signal power measured at range $r$ due to laser emission at $\lambda_{L}$ using a bandpass centered at $\lambda_{H}$ with bandpass width $\Delta \lambda_{H}$.

2. $P_{0}$ is the laser power.

3. $c \tau / 2$ is one half of the length of the volume illuminated by the laser beam with $\tau$ being the laser pulse temporal width and $c$ the speed of light.

4. $A / r^{2}$ is the solid angle subtended by the receiver as viewed from the scattering volume.

5. $\eta\left(\lambda_{H}\right)$ is the optical efficiency of the water vapor channel evaluated at the bandpass center wavelength (CWL) of $\lambda_{H}$.

6. $F_{H}(T)$ is the factor in the lidar equation that carries all the temperature dependence of the Raman water vapor measurement and is defined as 


$$
F_{H}(T)=\frac{\int_{\Delta \lambda_{H}} \eta\left(\lambda^{\prime}\right) d \sigma_{H}\left(\lambda^{\prime}, \pi, T\right) / d \Omega \mathrm{d}\left(\lambda^{\prime}\right)}{\eta\left(\lambda_{H}\right) d \sigma_{H}(\pi) / d \Omega}
$$

where $d \sigma_{H}(\pi) / d \Omega$ is the full Raman backscatter cross section, here restricted to the $Q$-branch due to the width of the filter considered, when excited at the laser wavelength. $d \sigma_{H}\left(\lambda^{\prime}, \pi, T\right) / d \Omega$ is the temperaturedependent Raman backscatter cross section where we note that the individual lines within the $Q$-branch are temperature dependent but the integral over the full $Q$-branch, given by $d \sigma_{H}(\pi) / d \Omega$, is essentially temperature independent. $\eta\left(\lambda^{\prime}\right)$ is the optical efficiency of the channel passband as a function of wavelength, $\lambda^{\prime}$, within the interval $\Delta \lambda_{H}$ that is defined by the filter passband. In the numerical work to follow, the integral in the numerator is implemented as a simple summation [20] over the lines tabulated in Avila et al. [21] $F_{H}$ provides the fraction of the full Raman $Q$-branch cross section that is transmitted by the Raman lidar channel passband (e.g., interference filter) given the atmospheric temperature of the scattering volume and the transmission characteristics of the passband.

7. $\beta_{\pi}^{H}\left(\lambda_{L}, r\right)=N_{H}(r) d \sigma_{H}(\pi) / d \Omega$ is the Raman backscatter cross section due to excitation, at the laser wavelength, of $N_{H}$ concentration of water vapor molecules at range $r$.

8. $\exp \left(-\int_{0}^{r}\left[\alpha\left(\lambda_{L}, r^{\prime}\right)+\alpha\left(\lambda_{H}, r^{\prime}\right) \mathrm{d} r^{\prime}\right]\right)$ is the roundtrip transmission to the scattering volume and back and involves the integral of the extinction, $\alpha$, at the laser wavelength on the outbound path and at the Raman shifted wavelength on the return path.

9. $B\left(r, \lambda_{H}, \Delta \lambda_{H}\right)$ is the background power due to skylight and detector noise.

It is the $F_{H}$ term defined in number 6 above that will receive much of the focus of the following sections.

\section{Simulations of the Raman Water Vapor Spectrum}

The temperature-dependent correction discussed here has been applied in different contexts. It has been used to improve the accuracy of water vapor data acquired during field campaigns, such as the International $\mathrm{H}_{2} \mathrm{O}$ Project [4], the AIRS Water Vapor Experiment-Ground [18], the Water Vapor Experiment Satellite/Sondes experiment [6], and for extended meteorological observations in Japan [22]. It has also been used as part of the implementation of an independent calibration of a Raman water vapor lidar [23,24]. All of these applications have been based on the tripled Nd:YAG laser $(\sim 354.7 \mathrm{~nm})$ and that is also the focus of this article.

It was noted before [4] that, for a laser output wavelength of $354.7 \mathrm{~nm}$, tuning a $0.25 \mathrm{~nm}$ wide interference filter to a wavelength $\sim 407.45 \mathrm{~nm}$ (Raman shift of $\sim 3649 \mathrm{~cm}^{-1}$ ) would minimize the temperature dependence of the Raman signal from water vapor although the exact temperature-independent location in the spectrum is a function of the filter shape. Also, with typical uncertainties in the CWL of the interference filter in the range of $0.01-0.05 \mathrm{~nm}$, it becomes difficult to tune an interference filter with confidence to either minimize the temperature dependence or to maximize the signal intensity, where we note that signal intensity is sacrificed if the filter is tuned to the nearly temperature insensitive point of $\sim 3649 \mathrm{~cm}^{-1}$. We will illustrate this after first demonstrating calculations of the Raman $Q$-branch using the data provided by Avila et al. [20].

Avila et al. 2004 [20] is an update to the 1999 work of the same Spanish group. The main advances in the 2004 work are due to improved ro-vibrational wavefunctions and are seen at temperatures exceeding $400 \mathrm{~K}$ and regions outside of the $Q$-branch. To illustrate the use of the published transitions, the Raman $Q$-branch was simulated at $297 \mathrm{~K}$ with $0.14 \mathrm{~cm}^{-1}$ resolution and is shown in Fig. 1. Two filters with approximate widths of $0.25 \mathrm{~nm}$ (the first from Fig. 3 of Whiteman et al. [4] and here tuned to $3652 \mathrm{~cm}^{-1}$, the second to be described later) are also shown for comparison purposes. The first of these will be the spectral shape used for most of the calculations to follow. The $Q$-branch spectrum shown in Fig. 1 compares favorably with Fig. 4(b) of Avila et a $\bar{l}$. 2004 [20] and is essentially indistinguishable from the spectrum calculated using the results from Avila et al.1999 [11] (Fig. 1 in [16]). The calculation of the full Raman $Q$-branch cross section performed here also agrees to within $0.001 \%$ of the values shown in Table 3 of Avila et al. 2004 [20] when using the same emission wavenumber and partition function values [25]. It should be noted that, based on theoretical considerations and confirmed through calculations using these 2004 data, the full $Q$-branch intensity is essentially independent of temperature over the range of temperatures of interest in Raman lidar measurements of the normal atmosphere.

We now focus on calculating the $F_{H}$ term shown in Eq. (1) as this term carries all the temperature dependence of the Raman lidar equation. As mentioned, $F_{H}$ can be considered as the effective Raman $Q$-branch cross section that results from

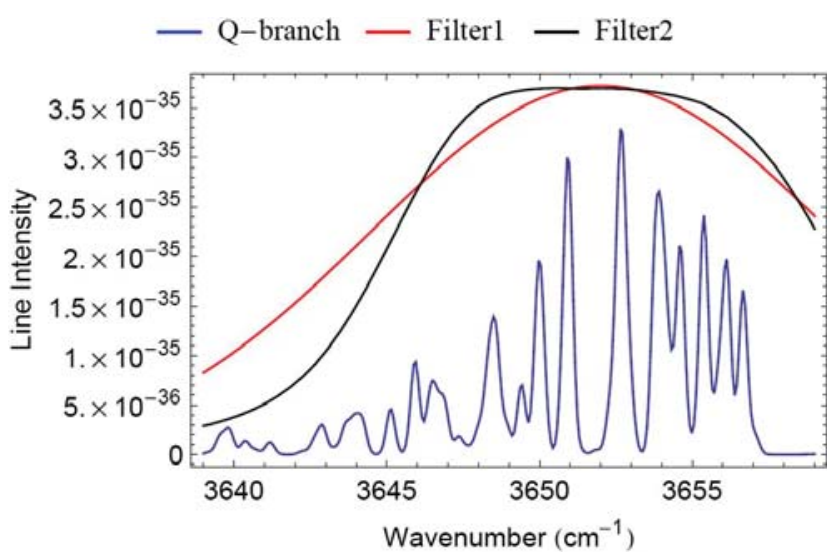

Fig. 1. Calculation of the Raman water vapor $Q$-branch simulated with $0.14 \mathrm{~cm}^{-1}$ resolution at $297 \mathrm{~K}$ in order to replicate Fig. 4(b) in Avila et al. 2004 [20]. Two filter shapes are shown corresponding to the filters used in experiments performed in 2007 and 2011, respectively, and are described later in the text. 


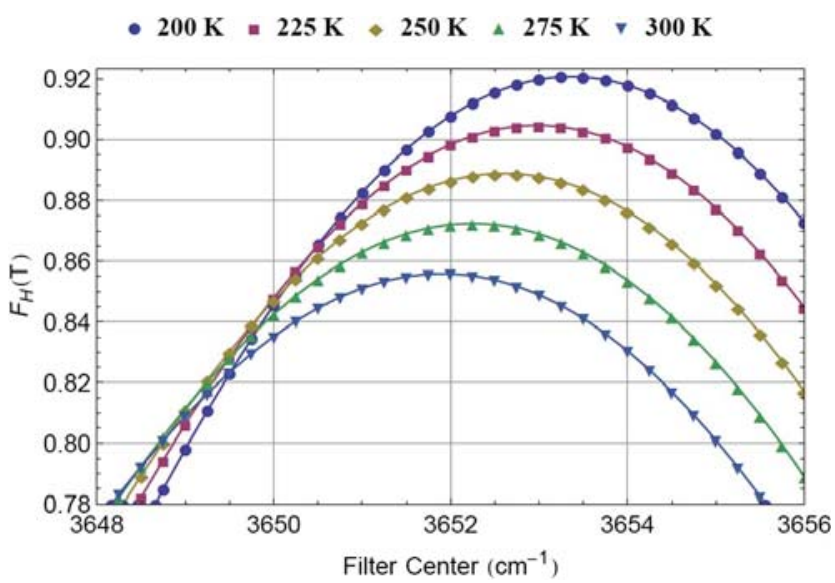

Fig. 2. Calculation of $F_{H}$ for Filter1 shown in Fig. 1 but with the center at different positions in the water vapor $Q$-branch. Calculations are performed over the range of 200-300 K. The peak in the $F_{H}$ curve shifts approximately $1 \mathrm{~cm}^{-1}$ over the $100 \mathrm{~K}$ range considered. Note that a filter located at a shift of $3649.5 \mathrm{~cm}^{-1} \mathrm{lim}$ its the temperature sensitivity to approximately $1 \%$ over the range of temperatures considered, but that the transmitted intensity for a filter positioned at this location is $\sim 4-12 \%$ less than if the filter were positioned at the peak of the $F_{H}$ curve.

the convolution of the individual temperaturedependent lines in the $Q$-branch spectrum and the spectral passband in use. Figure 2 shows calculations of $F_{H}$ using the shape of Filter1 shown in Fig. 1 where that shape is centered over a range of $3648-3656 \mathrm{~cm}^{-1}$, simulating a tilt-tuning experiment. Temperatures between 200 and $300 \mathrm{~K}$ are considered given that this range covers most temperatures encountered in the troposphere and lower stratosphere. The largest effective cross sections are achieved at CWL positions between approximately 3652 and $3653 \mathrm{~cm}^{-1}$ depending on temperature. This amounts to a total shift of approximately $1 \mathrm{~cm}^{-1}$ in the peak of the $F_{H}$ curve over the $100 \mathrm{~K}$ temperature range considered. The figure indicates that at an atmospheric temperature of $300 \mathrm{~K}$, using Filter 1

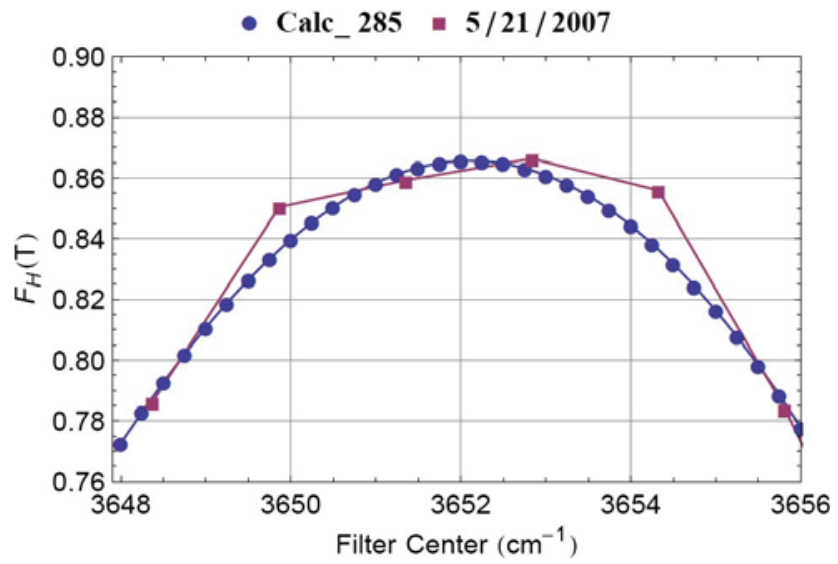

Fig. 3. (Blue) Theoretical calculation of $F_{H}$ (T) using Filter1 shown in Fig. 1 for a temperature of $285 \mathrm{~K}$ (Media 1). (Magenta) Tilt-tuning lidār measurements from the night of 21 May 2007 of transmitted intensity in the lidar water vapor channel as a function of tilt angle normalized to the theoretical $F_{H}(\mathrm{~T})$ curve.
- Calc_278 K $=10 / 31 / 2011 \mathrm{a}-10 / 31 / 2011 \mathrm{~b}$

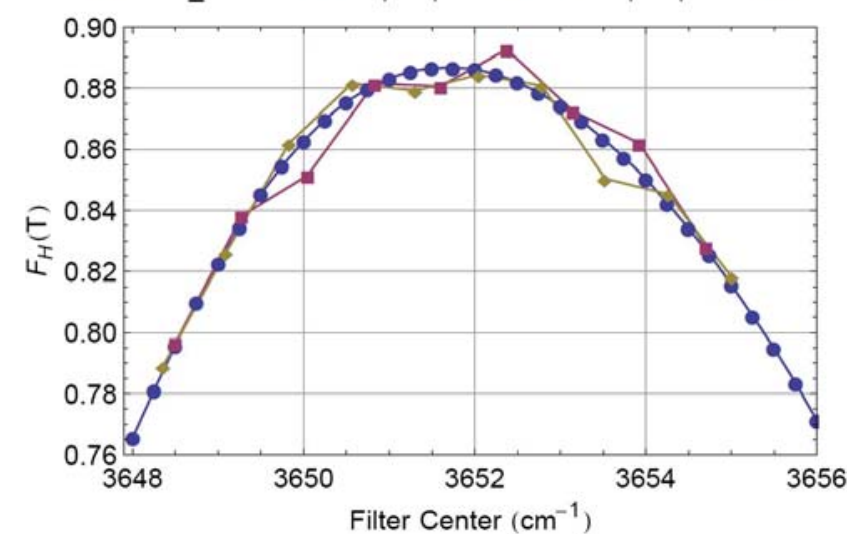

Fig. 4. (Blue) Theoretical calculation of $F_{H}(\mathrm{~T})$ using Filter2 shown in Fig. 1 for a temperature of $278 \mathrm{~K}$. (Magenta and Brown) Lidarderived results from two similar tuning experiments performed on 31 October 2011 using the tilt module described in the text.

shown in Fig. 1 but considering it to have $100 \%$ peak transmission, almost $86 \%$ of the total Raman $Q$-branch cross section would be transmitted. Considering a $200 \mathrm{~K}$ temperature, the same filter positioned at $3653 \mathrm{~cm}^{-1}$ would transmit approximately $92 \%$ of the Raman $Q$-branch, an increase of more approximately $7 \%$. The plot also indicates that, for this filter, the transmitted intensity of the filter positioned at $3649.5 \mathrm{~cm}^{-1}$ would change by approximately $1 \%$ over the $100 \mathrm{~K}$ range considered. Positioning the filter passband at this location is the approach that has been taken by some groups [8]. But there are several reasons to prefer positioning the passband to the peak of the $F_{H}$ curve.

As shown in Fig. 2, depending on temperature, positioning the filter to the nearly temperatureinsensitive point of $3649.5 \mathrm{~cm}^{-1}$ sacrifices between $4 \%-12 \%$ of the transmitted intensity versus positioning the filter at the peak of the $F_{H}$ curve. Positioning the filter at the peak of $F_{H}$ introduces additional temperature dependence when compared to positioning the filter at $\sim 3649.5 \mathrm{~cm}^{-1}$ but, as will be shown, performing an accurate correction for this temperature dependence requires only an approximate knowledge of the atmospheric temperature profile. Finally, positioning the filter to $3649.5 \mathrm{~cm}^{-1}$, which is along the shoulder of the $F_{H}$ curve, is more difficult experimentally than positioning the filter to the peak of the $F_{H}$ curve.

\section{Tilt-Tuning to the Maximum in the $\boldsymbol{F}_{H}$ Curve}

The procedure suggested here is to acquire successive lidar profiles through sequential tilt-tuning of the filter (or scanning the output of a spectrometer) and comparing these results with calculated $F_{H}$ curves as shown in Fig. 2. This is the procedure that we have followed since first assessing the temperature correction for the IHOP [4] and AWEX-G [18] field campaigns, held in 2002 and 2003, respectively, that were mentioned earlier. During the early implementation of this technique, we used wedges of varying thicknesses to implement different angular 
tilts of a mounted interference filter. At each angle, a separate lidar profile was acquired. This procedure required dismounting the filter between each lidar profile, which was a time consuming process. Nonetheless, under stable atmospheric conditions this approach provided reasonable results. An example of this is shown in Fig. 3 . The theoretical $F_{H}$ curve, calculated using Filter 1 shown in Fig. 1 for a temperature of $285 \mathrm{~K}$ (see Fig. $\underline{3}$ multimedia file Media 1 for a pdf of the Mathematica notebook used to calculate this), is shown along with the results of a tilt-tuning lidar experiment performed on the night of 21 May 2007. The temperature of $285 \mathrm{~K}$ was chosen to reflect the approximate temperature of the lower troposphere during the time of the lidar measurements on this evening. To perform the lidar measurements, the water vapor filter was sequentially tilted in 1 degree increments by using wedges of appropriate thicknesses. At each angle, water vapor profiles were acquired under stable nighttime conditions. To create the lidar derived values shown in Fig. 3, the sum of the counts in the water vapor channel between the altitude ranges of approximately $0.5-2.0 \mathrm{~km}$ was formed for each profile. A best fit of these intensity values to the $F_{H}$ values shown was done to find the correspondence between tilt angle and Raman shift as shown in the figure. As the figure indicates, a 1 degree tilt in the filter corresponded to a change in filter spectral location of approximately $1.5 \mathrm{~cm}^{-1}$. The agreement between the calculated curve and the 2007 measurements is quite good particularly in the shoulders. The close agreement of the shoulders permits the center of the filter to be determined with an estimated uncertainty of less than $0.2 \mathrm{~cm}^{-1}$.

Since 2010, we have used specially designed filter modules for both the water vapor and nitrogen channels in the ALVICE Raman lidar. These modules permit the tilt-tuning experiment to be quickly and repeatedly implemented. The filter modules also permit accurate changes in tilt angle of less than 0.1 degrees improving on the ability to assess the center location of the filter over the 2007 experiment shown in Fig. 3. Two experiments performed on the night of 31 October 2011 using the tilt module are shown in Fig. $\underline{4}$ where the $F_{H}$ curve shown was calculated at the temperature of $278 \mathrm{~K}$, the mean temperature on this night of the atmospheric layer used for the lidar measurements. Note that Filter2 from Fig. 1 was used for these 2011 experiments. Most of the differences in the $F_{H}$ curves between Figs. $\underline{3}$ and $\underline{4}$ are due to the difference in filter shape as the atmospheric temperature difference of $7 \mathrm{~K}$ does not produce a large change in the shape of the $F_{H}$ curve as can be discerned from Fig. 2 . Performing the tilttuning experiment in a short period of time in general decreases the influence of changing atmospheric water vapor amounts on the results. The ability to acquire data in a shorter period of time, along with the higher angular resolution of the 2011 measurements, helps to explain the better correspondence between the theoretical curve and the measurements taken in 2011 versus those in 2007 shown in Fig. 3. This better agreement of curves reduces the uncertainty in the assignment of the center position of the filter to an estimated $0.1 \mathrm{~cm}^{-1}$.

Knowledge of the atmospheric temperature is needed to determine the spectral location of the interference filter through the experiments illustrated in Figs. $\underline{3}$ and $\underline{4}$. Note, however, that the peaks of the intensity curves shown in Fig. 2 change by only $\sim 1 \mathrm{~cm}^{-1}$ over the $100 \mathrm{~K}$ range considered. Therefore, only an approximate knowledge of the temperature of the atmospheric layer used for the summation of the lidar measurements is needed in order to determine, with high accuracy, the appropriate $F_{H}$ curve to use for fitting the lidar-derived data. This is summarized in Table 1 where a $25 \mathrm{~K}$ change in the temperature of the atmospheric layer used for lidar measurements of signal intensity implies approximately a $0.7 \mathrm{~cm}^{-1}$ change in the location of the peak in the $F_{H}$ curve. Under most experimental conditions the knowledge of the mean temperature of the atmospheric layer probed by the lidar can be determined to much better than $25 \mathrm{~K}$. For example, if the mean temperature of the atmospheric layer probed is known to within $3 \mathrm{~K}$, the uncertainty in the theoretical position of the peak of the $F_{H}$ curve in comparisons, such as shown in Figs $\underline{3}$ and $\underline{4}$ can be limited to less than $0.1 \mathrm{~cm}^{-1}$ which corresponds to approximately $0.001 \mathrm{~nm}$ when considering Raman lidar measurements of water vapor using the tripled $\mathrm{Nd}$ :YAG laser $(\sim 354.7 \mathrm{~nm})$.

\section{Application of the Temperature Correction}

The temperature correction is determined by evaluating $F_{H}$ as a function of altitude and including $F_{H}$ as a multiplier in the lidar equation as shown in Eq. (1). Given the ability to evaluate $F_{H}$ for a single temperature, it is straightforward to evaluate $F_{H}$ for the entire lidar profile. But given that $F_{H}$ changes by $6 \%-9 \%$ over a $100 \mathrm{~K}$ range for the filter configurations considered in Table 1, it can be seen that the $F_{H}$ correction is not strongly a function of temperature. To illustrate this point, consider Fig. 5 .

Figure 5 presents $F_{H}$ versus altitude for the situation of Filter1 shown in Fig. 1 centered at $3652 \mathrm{~cm}^{-1}$, a situation similar to that achieved in the tilt-tuning calibration of the Raman lidar in the ALVICE system

Table 1 Considering the $0.25 \mathrm{~nm}$ Filter Shown in Fig. 1, the Peak in $F_{H}$ as a Function of Filter Spectral Location; Percentage Change in $F_{H}$ over the $100 \mathrm{~K}$ Range of $200-300 \mathrm{~K}$ is Given

Peak of $F_{H}$ Curve as a Function of Atmospheric Layer and Percent Change in $F_{H}$ Over Range of Atmospheric Temperatures

\begin{tabular}{ccc}
\hline $\begin{array}{c}\text { Atmospheric } \\
\text { Temperature (K) }\end{array}$ & $\begin{array}{c}F_{H} \text { Peak Intensity } \\
\left(\mathrm{cm}^{-1}\right)\end{array}$ & $\begin{array}{c}\text { Change }(\%) \text { in } F_{H} \\
\text { from 200 to 300 K }\end{array}$ \\
\hline 200 & 3653.2 & 8.9 \\
225 & 3652.9 & 8.4 \\
250 & 3652.6 & 7.2 \\
275 & 3652.3 & 6.7 \\
300 & 3652 & 6.1 \\
\hline
\end{tabular}




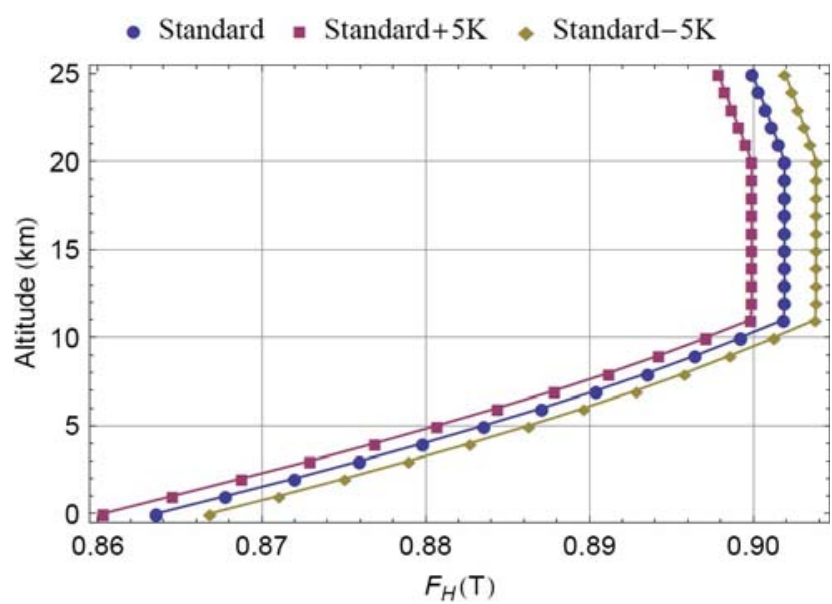

Fig. 5. $\quad F_{H}$ versus altitude for the U.S. Standard Atmosphere [26] plus and minus $5 \mathrm{~K}$ using Filter1 shown in Fig. 1 . Note that to constrain the uncertainty in the temperature correction to less than $0.4 \%$, the atmospheric temperature at a given altitude need not be known to better than approximately $\pm 5 \mathrm{~K}$.

when the focus is on lower tropospheric measurements. The U. S. Standard Atmosphere [26] is used for the temperature profile and deviations of $\pm 5 \mathrm{~K}$ are considered at each altitude. Over the range of temperatures in the U. S. Standard Atmosphere from the surface to the tropopause, $F_{H}$ changes from approximately 0.863 to 0.902 for a total change of $4.3 \%$. For temperature changes of $\pm 5 \mathrm{~K}$ considered at each altitude, the values of $F_{H}$ deviate by less than $0.2 \%-0.4 \%$. Therefore, the figure illustrates that, provided the temperature is known to within $\pm 5 \mathrm{~K}$ as a function of altitude, the uncertainty in the $F_{H}$ value due to uncertainties in atmospheric temperature can be constrained to less than $0.4 \%$.

\section{Discussion}

Performing the lidar tilt-tuning experiment and performing a best-fit of the lidar-derived values to the theoretically determined ones as shown in Figs. $\underline{3}$ and $\underline{4}$ must be done with care. Note that the entire range of $F_{H}$ intensity values plotted in the figures is approximately $10 \%$. Therefore, any changes in atmospheric water vapor amounts or laser output power during the time of the tilt-tuning experiment should be determined to much better than $10 \%$. A sequence of lidar profile measurements of water vapor mixing ratio in the nominal configuration, taken both before and after the tilt-tuning experiment, can be used to assess the stability of the water vapor field during the experiment. Correlative measurements of total column water can also help to assess changes in water vapor amount during the experiment. Performing the experiment quickly, as is aided by the ability to tilt-tune the filter in place, also helps to minimize changes in water vapor amount. Assuming insignificant changes in the atmospheric density and extinction profiles during the experiment, changes in laser power are compensated for in the lidar measurements used to determine the mixing ratio. Note, however, that for the measurements shown in Figs. 3 and 4 only the water vapor signal intensity was monitored and not the mixing ratio. The stability of the measurements was such, however, that no adjustments were needed for changes in water vapor concentration or in laser power.

As mentioned earlier, our experience at GSFC is that wavelength uncertainties in manufacturer's transmission curves are typically $0.01-0.02 \mathrm{~nm}$. Also, the laser output wavelength changes as the atmospheric index of refraction changes due to changes in temperature and pressure [27]. One of the consequences of this is that there is a small change in wavelength as a function of pressure at a given elevation even if the temperature is held constant. If the elevation at which the laser is operated changes, larger changes in wavelength can occur. For example, the tripled Nd:YAG laser operated at an elevation of $2.5 \mathrm{~km}$ will have an output wavelength approximately $0.027 \mathrm{~nm}$ longer than if operated at sea level if only pressure changes are considered. Such a change in wavelength, if uncompensated for, and assuming a $0.25 \mathrm{~nm}$ filter can alter the water vapor mixing ratio calibration value of a Raman lidar by $2 \%-4 \%$ depending on the CWL of the filter. Because of these changes in laser output wavelength as a function of atmospheric refractive index, careful estimates of the actual laser output wavelength include uncertainties in the range of 0.01-0.02 $\mathrm{nm}$ [7]. A reasonable estimate for the typical combined uncertainty of the relative spectral locations of the interference filter and the laser output wavelength would seem, therefore, to be in the range of $0.01-0.04 \mathrm{~nm}$, although larger errors in the filter CWL would increase this range. The range of $0.01-0.04 \mathrm{~nm}$ corresponds to approximately $1-3 \mathrm{~cm}^{-1}$ when considering use of the tripled Nd:YAG laser. For the filters of $\sim 0.25 \mathrm{~nm}$ width considered here, this uncertainty in the spectral location of the CWL of the filter translates into an uncertainty in the required temperature correction over a $100 \mathrm{~K}$ range of up to $\sim 3 \%$ (refer to Fig. $\underline{4}$ in [16]). By comparison, as described above, the combined uncertainty of the relative location of the interference filter with respect to the laser output wavelength is determined by the uncertainty of the atmospheric layer temperature and the uncertainty of the fitting of the theoretical and measured curves as demonstrated in Figs. 3 and 4 . Each of these uncertainties was estimated to not exceed $0.1-0.2 \mathrm{~cm}^{-1}$. This is approximately an order of magnitude lower uncertainty than that achieved by normal laboratory procedures. This lower uncertainty in the filter spectral location limits the uncertainty in the required temperature correction to $\sim 0.25 \%$ at a given atmospheric pressure. Pressure changes of $25-50 \mathrm{hPa}$ in the laboratory environment where the laser is housed do not significantly increase this uncertainty estimate. Also, it should be noted that this low uncertainty in the spectral location of the channel passband translates into high stability of calibration. With the ability to align the interference filter with the spectral passband with such accuracy, repeated 
experiments as described here can help to maintain calibration even if temperature, mechanical disturbance or aging produces changes in the center of the passband [10]. Repeated experiments of this type also help to randomize a component of the systematic error budget of the lidar which is advised for developing time series of water vapor data for trend studies $[28,29]$. For a mobile system, such as the ALVICE laboratory, where measurements may occur at varying altitudes or where mechanical disturbances may occur in transit, it is advisable to perform the tuning procedure described here at each location where measurements are taken.

As stated previously, Fig. 2 indicates that, for the filter considered, selection of a filter center passband of $3649.5 \mathrm{~cm}^{-1}$ reduces the temperature sensitivity of $F_{H}$ to approximately $1 \%$ over the $100 \mathrm{~K}$ range considered. But this sacrifices between $4 \%-12 \%$ of potential signal intensity when compared to tuning the interference filter to the peak of the $F_{H}$ curve. An application of Raman lidar focused on boundary layer meteorology or convective storm development, such as the IHOP experiment in 2002 [30] could benefit from placement of the interference filter at a center location of $3652 \mathrm{~cm}^{-1}$. Whereas, an experiment focused on upper tropospheric ice supersaturation in the tropics or trend studies could well benefit from a placement of the center of the filter at $3653.2 \mathrm{~cm}^{-1}$ since this placement of the filter would result in approximately $10 \%$ more water vapor signal strength for the cold upper tropospheric measurements as compared with those that would be taken if the filter were placed at $3649.5 \mathrm{~cm}^{-1}$. With the techniques outlined here, either of these peak spectral locations could be achieved with accuracy of $\sim 0.1 \mathrm{~cm}^{-1}$ ensuring that the temperature correction needed for either filter position could be accurately applied.

Figure $\underline{5}$ demonstrates that knowledge of the atmospheric temperature profile to within $\pm 5 \mathrm{~K}$ can limit the uncertainty of the applied temperature correction, due to uncertainties in atmospheric temperatures, to approximately $0.2 \%-0.4 \%$. When combined with the uncertainty in the temperature correction due to uncertainty in spectral filter location, the propagated uncertainty in the temperature correction becomes approximately $0.5 \%$. If a systematic bias of $0.5 \%$ can be considered insignificant for a given application, then an approximate temperature profile can be used for a sufficiently accurate application of the temperature dependence correction. If, for example, the temperature correction needs to be applied over a $24 \mathrm{~h}$ dataset, it may be necessary to use 2 or even 3 approximate temperature profiles during a $24 \mathrm{~h}$ period, where an interpolation can be done between the 2 or 3 profiles, to increase the accuracy of the correction. Such an approach can be used to limit the uncertainty of the temperature correction to less than $0.5 \%$ even for data acquired over an extended period under day and night conditions.

Correction of systematic bias is a required component of good metrological practice [31] requiring a sound estimate of the additional uncertainty in the final data product that is created by applying any corrections. Because it results in higher signal strength and allows for a more accurate spectral positioning of the interference filter, the case being made here is to tune the Raman lidar water vapor channel to a point that maximizes signal strength but also that creates a small temperature dependent bias. The bias can then be corrected, using the procedures outlined here, where the additional uncertainty due to the correction is then included in the uncertainty budget of the data. Considering together an uncertainty in spectral location of the filter and an uncertainty in atmospheric temperature of $5 \mathrm{~K}$ as a function of altitude, the total uncertainty in the temperature correction determined here remained below $0.5 \%$. This is the additional uncertainty due to the temperature correction. Given that Raman lidar random uncertainties are typically in the 5\%-10\% range or greater and that calibration uncertainty is approximately 5\% or greater [24,32], an increase of $0.5 \%$ in the total uncertainty budget would seem insignificant for most applications of Raman water vapor lidar data. If lower uncertainty in the temperature correction is needed, this can be achieved by a finer resolution fitting of lidar measurements to the theoretical $F_{H}$ curve (Figs. $\underline{3}$ and $\underline{4}$ ) and by using a more accurate profile of atmospheric temperature.

\section{Summary and Conclusions}

Previous efforts to calculate the temperature dependence of the Raman water vapor lidar spectrum for use in Raman lidar profiling of water vapor have required very accurate characterization of both the output laser wavelength and spectral passband characteristics of the water vapor channel. Typical accuracies in the determination of these two quantities result in an uncertainty in the temperature dependence of the Raman water vapor lidar measurement of up to $3 \%$. We have described here an experimental technique that evaluates the convolution of the output laser wavelength and the spectral passband characteristics together with a resultant accuracy that greatly exceeds that of typical laboratory results. The technique involves performing atmospheric profiling with a sequentially tilted interference filter to evaluate the curve of $F_{H}$ as a function of tilt angle. It is not necessary to know the effective index of the interference filter; it is only necessary to be able to discern a clear peak in the curve. The peak in this curve along with an approximate knowledge of atmospheric temperature permits the peak of the interference filter transmission curve to be determined with accuracy of $\sim 0.1 \mathrm{~cm}^{-1}$. By positioning the filter at the peak in this intensity curve, maximum transmitted intensity at that temperature is achieved although a correction is needed for the temperature dependence of the measurement. Depending on whether the Raman lidar is optimized for lower tropospheric or upper tropospheric measurements, the magnitude of the temperature 
correction ranges between 6\%-9\% for a $100 \mathrm{~K}$ change in atmospheric temperatures. Under many circumstances the range of temperatures from the surface to the tropopause will be less than $100 \mathrm{~K}$ indicating that the magnitude of the correction is less, as was the case illustrated in Fig. 5. It was also shown that only an approximate knowledge of the actual atmospheric temperature profile is needed to limit uncertainties in the correction to typically less than $0.5 \%$. This increase in uncertainty is likely tolerable for any application of Raman water vapor lidar measurements. It is our hope that the procedure outlined here will ease the efforts to provide accurate narrowband Raman water vapor lidar measurements. A Mathematica notebook that demonstrates the calculations used here is available from the lead author.

\section{Appendix: A}

The filters used in this study possessed CWL approximately $0.05 \mathrm{~nm}$ longer than the peak of the water vapor $Q$-branch (e.g., see Fig. 3 in [4]]). Because of this, it was possible to scan the full set of wavenumbers shown in the figures in this paper. If, on the other hand, the CWL of the filter when positioned normal to the optical path is closer to the peak of the water vapor spectrum, it may not be possible to create a single-peaked $F_{H}$ curve as shown in Figs. $2-\underline{4}$. For example, consider the same Filter 1 from Fig. 1 above but with the center of the filter, when normal to the optical path, at $3653 \mathrm{~cm}^{-1}$. With such a filter, wavenumbers larger than 3653 cannot be reached through a tilt-tuning exercise since tilting an interference filter from the normal position causes the transmitted wavelength to decrease. The type of $F_{H}$ curve that would result using such a filter is illustrated in Fig. $\underline{6}$. The same fitting exercise described in the paper can be done to determine spectral location of the filter but the shapes of the curves will be quite different. Note that this effect does exist for experiments based on scanning the output of a spectrometer.

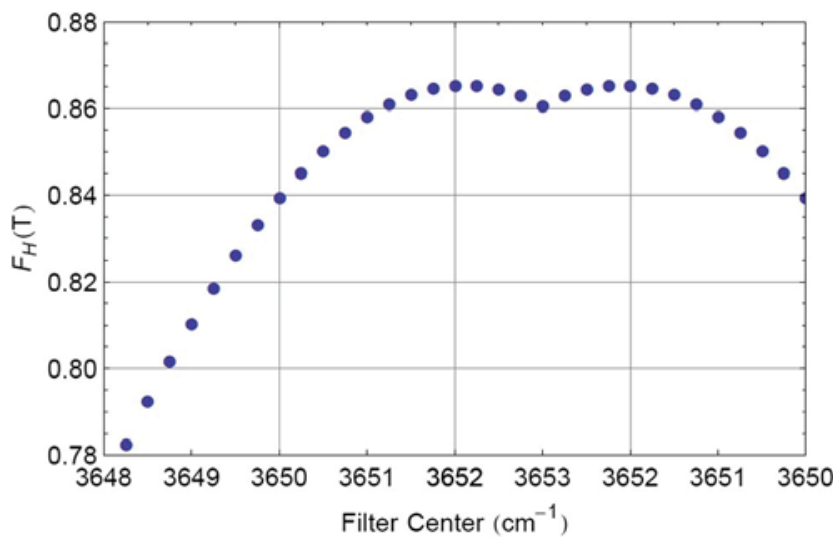

Fig. 6. $\quad F_{H}$ versus wavenumber plot that results for a tilting experiment using a $0.25 \mathrm{~nm}$ filter with passband centered at $3653 \mathrm{~cm}^{-1}$ when normal to the optical path. Note that no wavenumbers above 3653 can be reached through the filter tilting experiment.
We thank Dr. Jose María Fernández for fruitful discussions regarding the absolute accuracy of the Avila et al. 2004 [20] cross section values. The experimental technique of determining the $F_{H}$ curve through tilt-tuning of interference filters was developed as part of the NASA/Earth Science Technology Office funded Advanced Component Technology Program involving Barr Associates and NASA/GSFC. This effort has also been supported by the NASA Atmospheric Composition Program, managed by Ken Jucks. The mention of a particular manufacturer does not constitute an endorsement by NASA.

\section{References}

1. J. Cooney, "Measurement of the ratio of the vibrational Raman cross section for $\mathrm{H}_{2} \mathrm{O}$ vapor to the nitrogen vibrationalrotational Raman band," Spectrosc. Lett. 3, 305-309 (1970).

2. S. H. Melfi, "Observation of Raman scattering by water vapor in the atmosphere," Appl. Phys. Lett. 15, 295-297 (1969).

3. J. E. M. Goldsmith, F. H. Blair, S. E. Bisson, and D. D. Turner, "Turn-key Raman lidar for profiling atmospheric water vapor, clouds, and aerosols," Appl. Opt. 37, 4979-4990 (1998).

4. D. N. Whiteman, B. Demoz, P. Di Girolamo, J. Comer, I. Veselovskii, K. Evans, Z. Wang, M. Cadirola, K. Rush, G. Schwemmer, B. Gentry, S. H. Melfi, B. Mielke, D. Venable, and T. Van Hove, "Raman water vapor lidar measurements during the international $\mathrm{H}_{2} \mathrm{O}$ project. I. Instrumentation and analysis techniques," J. Atmos. Ocean. Technol. 23, 157-169 (2006).

5. D. N. Whiteman, I. Veselovskii, M. Cadirola, K. Rush, J. Comer, J. Potter, and R. Tola, "Demonstration measurements of water vapor, cirrus clouds, and carbon dioxide using a high-performance Raman lidar,” J. Atmos. Ocean. Technol. 24, 1377-1388 (2007).

6. M. Adam, B. B. Demoz, D. N. Whiteman, D. D. Venable, E. Joseph, A. Gambacorta, J. Wei, M. W. Shephard, L. M. Miloshevich, C. D. Barnet, R. L. Herman, J. Fitzgibbon, and R. Connell, "Water vapor measurements by Howard University Raman lidar during the WAVES 2006 campaign," J. Atmos. Ocean. Technol. 27, 42-60 (2010).

7. J. Reichardt, U. Wandinger, V. Klein, I. Mattis, B. Hilber, and R. Begbie, "RAMSES: German meteorological service autonomous Raman lidar for water vapor, temperature, aerosol, and cloud measurements," Appl. Opt. 51, 8111-8131 (2012).

8. T. S. Dinoev, V. B. Simeonov, Y. F. Arshinov, S. M. Bobrovnikov, P. Ristori, B. Calpini, M. B. Parlange, and H. van den Bergh, "Raman lidar for meteorological observations, RALMO_part I: instrument description," Atmos. Meas. Tech. Discuss. 5, 6867-6914, (2012).

9. C. R. Philbrick and K. R. Mulik, "Application of Raman lidar to air quality measurements," Proc. SPIE 4035, 22-33 (2000).

10. V. Sherlock, A. Hauchecorne, and J. Lenoble, "Methodology for the independent calibration of Raman backscatter water vapor lidar systems," Appl. Opt. 38, 5816-5837 (1999).

11. G. Avila, J. M. Fernández, B. Mate, G. Tejeda, and S. Montero, "Ro-vibrational Raman cross sections of water vapor in the OH stretching region,” J. Mol. Spectrosc. 196, 77-92 (1999).

12. W. F. Murphy, "The ro-vibrational Raman spectrum of water vapor n1 and n3," J. Phys. B 36, 727-732 (1978).

13. W. F. Murphy, "Intensities of rotation and vibration-rotation Raman transitions in asymmetric top molecules," J. Raman Spectrosc. 11, 339-345 (1981).

14. J. M. Fernández-Sánchez and W. F. Murphy, "Raman scattering cross sections and polarizability derivatives of $\mathrm{H}_{2} \mathrm{~S}, \mathrm{D}_{2} \mathrm{~S}$, and HDS," J. Mol. Spectrosc. 156, 431-443 (1992).

15. J. M. Fernández-Sánchez and W. F. Murphy, “True and effective polarizability tensors for asymmetric-top molecules: the rotational Raman spectra of $\mathrm{H}_{2} \mathrm{~S}$ and $\mathrm{D}_{2} \mathrm{~S}$," J. Mol. Spectrosc. 156, 444-460 (1992). 
16. D. N. Whiteman, "Examination of the traditional Raman lidar technique. I. Evaluating the temperature-dependent lidar equations," Appl. Opt. 42, 2571-2592 (2003).

17. D. N. Whiteman, "Examination of the traditional Raman lidar technique. II. Evaluating the ratios for water vapor and aerosols," Appl. Opt. 42, 2593-2608 (2003).

18. D. N. Whiteman, F. Russo, L. Miloshevich, B. Demoz, Z. Wang, I. Veselovskii, H. Voemel, S. Hannon, B. Lesht, F. Schmidlin, A. Gambacorta, and C. Barnet, "Analysis of Raman lidar and radiosonde measurements from the AWEX-G field campaign and its relation to aqua validation," J. Geophys. Res. 111, D09S09 (2006).

19. M. Bass, ed., Handbook of Optics, 2nd ed. (Optical Society of America, 1995).

20. G. Avila, J. M. Fernández, G. Tojeda, and S. Montero, "Raman spectra and cross sections of $\mathrm{H}_{2} \mathrm{O}, \mathrm{D}_{2} \mathrm{O}$, and $\mathrm{HDO}$ in the $\mathrm{OH} /$ OD stretching regions," J. Mol. Spectrosc. 228, 38-65 (2004).

21. M. Adam, "Notes on temperature-dependent lidar equations," J. Atmos. Ocean. Technol. 26, 1021-1039 (2009).

22. T. Sakai, T. Nagai, M. Nakazato, T. Matsumura, N. Orikasa, and Y. Shoji, "Comparisons of Raman lidar measurements of tropospheric water vapor profiles with radiosondes, hygrometers on the meteorological observation tower, and GPS at Tsukuba, Japan," J. Atmos. Ocean. Technol. 24, 1407-1423, (2007).

23. E. Landulfo, R. F. Costa, A. S. Da Torres, F. J. S. Lopes, D. N. Whiteman, and D. D. Venable, "Raman water vapor lidar calibration,” Proc. SPIE 7479, 74790J (2009).

24. D. D. Venable, D. N. Whiteman, M. N. Calhoun, A. O. Dirisu, R. M. Connell, and E. Landulfo, "A lamp mapping technique for independent determination of the water vapor mixing ratio calibration factor for a Raman lidar system," Appl. Opt. 50, 4622-4632 (2011).
25. J. M. Fernández-Sánchez (private communication, 2013).

26. U. S. Standard Atmosphere, 1976 (U.S. Government Printing Office, 1976).

27. B. Edlén, "The refractive index of air," Metrologia 2, 71-80 (1966).

28. D. N. Whiteman, K. C. Vermeesch, L. D. Oman, and E. C. Weatherhead, "The relative importance of random error and observation frequency in detecting trends in upper tropospheric water vapor," J. Geophys. Res. 116, D21118 (2011).

29. D. N. Whiteman, M. Cadirola, D. Venable, M. Calhoun, L. Miloshevich, K. Vermeesch, L. Twigg, A. Dirisu, D. Hurst, E. Hall, A. Jordan, and H. Vömel, "Correction technique for Raman water vapor lidar signal-dependent bias and suitability for water vapor trend monitoring in the upper troposphere," Atmos. Meas. Tech. 5, 2893-2916 (2012).

30. B. Demoz, C. Flamant, T. Weckwerth, D. Whiteman, K. Evans, F. Fabry, P. Di Girolamo, D. Miller, B. Geerts, W. Brown, G. Schwemmer, B. Gentry, W. Feltz, and Z. Wang, "The dryline on 22 May 2002 during IHOP: convective scale measurements at the IHOP profiling site, part I," Mon. Weather Rev. 134, 294-310 (2006).

31. "JCGM/WG 1: evaluation of measurement data-guide to the expression of uncertainty in measurement, International Bureau of Weights and Measures/Bureau International des Poids et Mesures," http://www.bipm.org/ utils/ common/documents/ jcgm/JCGM_100_2008_E.pdf, Working Group 1 of the Joint Committee for Guides in Metrology, 2008.

32. T. Leblanc, I. S. McDermid, and T. D. Walsh, "Ground-based water vapor Raman lidar measurements up to the upper troposphere and lower stratosphere for long-term monitoring," Atmos. Meas. Tech. 5, 17-36 (2012). 